\title{
Chapter five - 'Pedagogic Strategies': a conceptual framework for effective parent and practitioner strategies when working with children under five
}

\section{By Penny Lawrence \& Tracy Gallagher}

(A version of this chapter was published in the journal Early Childhood Development and Care 2015 DOI 10.1080/03004430.2015.1028390]

In this chapter we explain a research project in which we identified adult pedagogic strategies; these are ways in which adults already engage to support children's learning effectively. The strategies themselves make a conceptual framework. The Pedagogic Strategies framework was first developed by parents and practitioners for working with children aged three to five years at Pen Green Centre (Whalley and Arnold, 1997). At Pen Green we have created several frameworks to support the development of practice. We focus on professional development rather than training as we see the introduction to these frameworks as part of an ongoing process of reflection and review. This chapter focuses on the 2013 revision of the adult pedagogic strategies framework to encompass work with children under the age of three.

\section{What is effective pedagogy?}

In their longitudinal study Researching Effective Pedagogy in the Early Years (REPEY) Siraj-Blatchford et al, (2002), sought to broaden the definition of pedagogy from 'the practice of teaching', to consider the environment and the process of play while retaining a core of instruction, 'Pedagogy refers to that set of instructional techniques and strategies which enable learning to take place and provide opportunities for the acquisition of knowledge, skills, attitudes and dispositions within a particular social and material context' (Siraj-Blatchford et.al., 2002, pg.28). Their research suggested that particular differences in philosophies or curriculum priorities were not the main contributing factors to educational performance, so a setting may be effective without it being related to one approach or another. There is, nevertheless, a case for considering the underpinning values that define pedagogical 
effectiveness beyond economic effectiveness or outcome measures (Weikart, 2000), 'Pedagogy needs explicitly to be seen to encompass a spirit of enquiry and professional dialogue about why we do what we do' (Kinney, 2005, pg.4). The Pedagogic Strategies project at Pen Green aimed to develop locally what Coles (2002) calls 'professional judgment'. We wanted a framework that would provide staff with opportunities to review their practice, consider the aspects of their work they do well and identify how to make improvements.

Internationally Bertram and Pascal (2002) reviewed the early years pedagogical approaches of 20 different countries. They found a substantial consensus on reciprocal interactional pedagogy, 'adopting a flexible range of teaching and learning strategies according to the needs of the children' (Bertram and Pascal, 2002, Section 3.6, pg.22). Within the Effective Early Learning Project (EEL), Pascal and Bertram (1997) drew on the work of Rogers (1983) to think about the ways in which, as adults, we interact with young children in order to facilitate their learning in dynamic relational processes. This latter work informed the Pedagogic Strategies research project with parents and practitioners that we carried out at Pen Green.

\section{What are Adult Pedagogic Strategies?}

Adult Pedagogic Strategies are how adults effectively support children's learning and development. They are not a "to do" check list. They are effective strategies that are embedded in our practice. Noticing them, reflecting on them, observing and recognising the children's learning and development enables us to work more effectively, and critically reflect on our own development as pedagogues (Carr, 2001). They complement and integrate our work with other frameworks, the Early Years Foundation Stage (DfE, 2012) and the Teacher's Standards for qualification and continuing professional development (National College for Teaching and Leadership, 2013) as EYPS, EYTs and Qualified Teachers in early years and primary.

\section{The pedagogy of parents and the local context}

The role of parents has been extensively researched and yet has developed relatively recently in terms of pedagogy. In a literature review of developmentally appropriate practice and play-based pedagogy, Walsh, Sproule, McGuinness, Trew and Ingram (2010) did not consider the potential of parents beyond a role limited to the time of 
transitions for the child, although they called for a more open-minded evidence-based approach to understand pedagogy in the early years. An exceptional case within the REPEY findings did indicate the potential of home-based pedagogy. It described a situation where the effectiveness of the practitioners in the setting was limited, yet the cognitive outcomes for the children were excellent,

it was less the staff's interventions and more the parents' proactive behaviour towards their children's learning in the embedded, cultural context of the home, that provided a good basis for sustained shared thinking [...] the parents prepared themselves to provide a 'potent home-based pedagogy' on a daily basis! (Siraj-Blatchford \& Sylva, 2004, pg.726).

There can be no presumption that it is the setting that improves the teaching potential of the parents. The cultural contexts for children's learning vary, as will the extent to which children may learn through observation of their parents and practitioners (Rogoff, Mosier, Mistry \& Goncu, 1993). The construct of parents having a 'natural pedagogy', rather than what may otherwise be referred to as sensitive interaction or a high level of engagement, remains to be explicitly validated and current understanding of it is limited (Sage $\underline{\&}$ Baldwin, 2012). Nevertheless, the value of working with parents' pedagogy is that it includes what the children learn within their home and within their culture. Local knowledge construction aligns with England's Department for Education (DfE) (2014a) call for educational communities to generate evidence of effective practice that is owned at the setting level. At a local level the Pedagogic Strategies project at Pen Green, had also identified 'Principles of Engagement with Under Threes' (Tait, 2007) established by parents and staff in our area.

\section{The Methodological Approach to the research}

\section{Phase 1 Methodology}

In 1997 we invited parents to take part in a small study, funded by the then Teacher Training Agency (now the National College of Teaching and Leadership), to consider the pedagogic strategies that both workers and parents were using (Whalley \& Arnold 1997). We began by introducing a group of parents to the work on Adult Style which 
was being used in the Effective Early Learning Project (EEL) (Pascal \& Bertram 1997).

At first the Pen Green workers and parents used the EEL framework to discuss what they were seeing on several video sequences of adult/child interactions. The children were aged three to five years old. Workers then spent time video-filming this group of parents, each with their own child, settling them into nursery and then supporting them in their play. The same children were then filmed with their Family Worker from the nursery. All the parents and workers met and looked at the video material together and subsequently began to reflect on and analyse what were effective teaching strategies that were being used. From this detailed analysis, we arrived at a framework of effective adult teaching strategies used by both parents and nursery staff.

\section{Phase 2 Methodology}

More than a decade after the original research the review of the pedagogic strategies was an opportunity for us to respond to the increased number of children below the age of three that we were now working with at Pen Green. Therefore in 2012-2013 a second working group of parents and staff was formed. We decided we would repeat the method we had used in 1997 filming parents each with their own children and then filming the same children with their Family Worker. The parents and workers would once again meet to reflect on and analyse the video clips.

\section{Participatory Approach}

The decision to use participatory research in case studies aligns with our commitment to working with families and family workers (Desforges $\underline{\&}$ Abouchaar, 2003; Langford, 2010; and Whalley et al, in press). More than a method, it is a decision about an entire approach to research (Kindon, Pain, $\underline{\&}$ Kesby, 2007). In order to research with people not on them (Heron \& Reason, 2001) within a socially constructivist perspective (Creswell, 2012) we deliberately sought parents from the setting to take a participatory role in meaning making in 'a participatory process concerned with developing practical knowing' (Reason \& Bradbury, 2008, pg.4) and 
the resulting selection of participants can be seen as a strength in terms of validity. They contributed their knowledge and their values (see ethics section).

\section{Participants}

Six mothers decided to participate after due consideration of the time commitment involved. The process of self-selection was also an ethical selection to ensure that the burden for participants would be manageable (BERA, 2011). They were not representative of individuals who have less inclination or availability to participate, however the participants did not withdraw from the study because of time issues, neither was the study too large or too expensive, it was therefore an effective selection procedure. There were 6 mothers out of 16 families, over a third of the families using the provision for children under three at the time (the Nest). We considered this to be a large enough number to inspire confidence in our findings. Sourcing data with our participants was more important than the number of participants.

The participants reviewed their initial discussions and through the group's own processes of quality control decided to ensure increased male participation in the study. There had been no fathers present in the discussions, although they had seen the video clips that had been sent home for viewing. The case studies were extended to include two additional families from which the fathers could participate more fully.

\section{The Case Study Approach}

The participants did not form a sample, but case studies. Valuable findings can be made from a small number of case studies and the generalisability can be increased by the strategic selection of cases to provide rich information. 'Random samples emphasizing representativeness will seldom be able to produce this kind of insight; it is more appropriate to select some few cases chosen for their validity' (Flyvbjerg, 2006, pg.13). The rationale for selecting a case study approach derives from the situated nature of our enquiry, 'its real world context' (Yin, 2014, pg.8). A case study also allows for the use of tacit knowledge by those who interpret the findings (Simons, 2009). Trustworthiness (Lincoln $\underline{\&}$ Guba, 1985) and authenticity (Guba $\underline{\&}$ 
Lincoln, 1989) are evidenced by confirming results with participants and through the high level of their involvement.

\section{Visual Analysis}

Video episodes provide for a multi-modal reading of the inter-actions between the children and adults. For example the modes of gesture and gaze as well as vocalisations are important for children's learning (Goodwyn, Acredolo \& Brown, 2000; Raikes et al., 2014; Topping et al., 2013) and the research team drew on the work of Flewitt $(2006)$ and Norris $(2004,2011)$ to interpret these.

Each child was video recorded by a practitioner or the researcher with his/her parent while involved in a learning experience, and was also recorded with his/her family worker. The research group met over a series of early evening sessions to discuss the short (three to five minute) episodes. Two stages of analysis were derived from visual anthropology (Collier, 2001). The first was an open viewing initial stage of the whole experience of the episode. Rather than starting with a list of themes or codes and then applying them, comments were noted in the open viewing, identifying images or critical sequences in the episode for the second stage, a detailed interpretation. This was an inductive process, drawn out of the observations. Our closeness to the visual material countered the risk of seeking to confirm pre-existing theories (Flyvjberg, 2006). The project employed appreciative enquiry, drawing value from what we saw and from each other's evaluations (Gergen, 2001; Reed, 2006). Themes emerged through dialogue and comparison between the video clips. The themes were reported back to the broader staff team and were developed into strategies in dedicated continuing professional development meetings. There had been more than one possible interpretation and way of structuring the strategies and this whole staff evaluation was a key stage in constructing and embedding the knowledge collectively in our practice (Kindon et al., 2007).

\section{Shared Code of Ethics}

The project complied with the British Educational Research Association ethical guidelines (BERA, 2011). As democratic a relationship as possible was sought between the researcher, staff and parents (Nolen \& Vander Putten, 2007) and using 
the project setting's own ethical policy. These were relational ethics in an on-going relationship with the participants. We shared concern for the children's learning and development and concern for each other's experience. We saw a range of reactive attitudes $^{1}$ (Strawson, 1962) which were meaningful to and demonstrated between all the participants in the study: the practitioner's consideration of how one family's response to a video episode may effect another family; the practitioner's reflection on her own practice; the researcher's awareness of her presence and the potential changes caused to the children and staff experience; the parents' responses to a close study of their child.

To be clear, the main and over-riding responsibility for the conduct of the project was the researchers'. However as a research group we were developing judgement not only of effective pedagogy, but also of how we conducted ethical relationships in our setting. Eshleman (2014) situates these judgments on principles within our practice, 'Their justification refers back to an account of the reactive attitudes and their role in personal relationships, not to some independent theoretical account of the conditions on being responsible' (online). Our developmental partnership (Easen et al., 1992) was within an ethical space for sharing values and evaluation of pedagogy. It demonstrated 'a particular attitude that leaves open the possibility for ethical reflection' (Ramaekers \& Suissa, 2011, pg.198), rather than a parenting intervention to instruct parents or for them to feel they ought to develop expert knowledge. Leading the research we were mindful of our diverse roles and knowledge bases, neither assuming nor intending that parents and practitioners were or ought to become the same.

Power issues were addressed by the distribution of decision-making. In addition to the researcher, practitioners recorded each other's practice and led their own contribution. The setting manager did not decide what the observations should include. Parents discussed and decided what and when to record in their interactions. For example they selected the time of day to record that allowed for the most typical inter-action between adult and child. At the interpretation stage the participants chose which clips to focus on. The parent of each child led the discussion and the researcher and team 
manager were conscious to facilitate all participants, at all staff leadership levels to have time within the meetings. In addition practitioners were able to give feedback within regular supervision with their line manager. In the second phase of the research we considered how we heard the parents' contributions, we decided to include more individual communication to check transcripts and exchange feedback through phone calls and email. The voice of the participants could therefore be heard both in the group and outside it to allow time and convenience for reflection on the study.

\section{Insert table here}

The Revised Adult Pedagogic Strategies (2013)

1. Subtle Intervention - The adult watches and listens to what the child is doing before intervening.

2. Linking Experiences - The adults are aware of the child's experience with other adults at home and in the setting.

3. Acknowledging - The adult acknowledges the child's presence, emotions and capability by:

-being physically close to them including using touch if appropriate to make contact (see also strategy 8).

- tuning into the child's facial expressions and vocal intonation, including playfulness and teasing.

4. Working with the Child's Initiative and Agency - The adult considers what the child is bringing to each interaction, checks out the child's meaning and gives the child time to respond or to question. The adult encourages the child's curiosity and ability to make choices including taking appropriate risks.

5. Adult Learning - The adult is committed to his/her own learning. S/he is open to play and learn alongside the child, encouraging new learning for both child and adult. 
6. Adult Attitudes - The adult is aware of the impact of his or her own attitudes and beliefs and how these might affect the child's learning.

7. Using Language The adult knows about the child's home vocabulary, offers new information to the child, including preparation for what is about to happen and describing what has just happened, and language to support the child's actions.

8. Using the Body - The adult affords learning experiences using the body ranging from using slight touch to whole body experiences if appropriate. The adult knows how individual babies and children like to be held, rocked and comforted.

\section{Findings}

\section{New Strategies}

There was a degree of continuity with the original strategies identified by the first research cohort in 1997, and two newly identified strategies emerged (see Table 1). For example the pre-existing first strategy, 'Subtle intervention: The adult watches and listens to what the child is doing before intervening', was also identified in 2013. There was some adaptation of original strategies. The team leader for children below three years valued what she called 'the synergy and synchrony between staff and between staff and parents'. The children's experience is with and between the adults who have awareness of each other, both within the same period of time as the child moves between adults in the setting, and asynchronously between days and between home and setting. Although the 'in setting' aspect of the child's experience may have been understood to be in place in the pre-existing strategies, it was not stated explicitly. The second strategy was revised to incorporate it, 'Linking Experiences: The adults are aware of the child's experience with other adults at home and in the setting', whereas before the links had been stated as between home and setting. 
The two new strategies that emerged in 2013 were 'acknowledging the child's presence' and 'using the body', an embodied pedagogy, with the following definitions:

\section{Acknowledging}

'The adult acknowledges the child's presence, emotions and capability by: being physically close to him/her including using touch if appropriate to make contact; tuning into the child's facial expressions and vocal intonation, including playfulness and teasing'. An example of acknowledging was when practitioner, Sarah, used speech, touch, gesture and gaze to let two children, Oscar and Polly each know that she is paying attention to them when they both wanted to use the same push-along trolley at the same time.

\section{Using the Body}

'The adult affords learning experiences using the body ranging from using slight touch to whole body experiences if appropriate. The adult knows how individual babies and children like to be held, rocked and comforted'. An example of using the body was when Dawn allowed Danny to recline back extending his full length to relax and read a book in her arms.

The following case study demonstrates the different pedagogic strategies used with a child, in the first example by his family worker and in the second example with his mother.

\section{Case Study of Dawn with Danny}

In the first part of the case study family worker, Dawn, interacts with Danny (28 months) using the following Adult Pedagogic Strategies: Subtle Intervention (1); Acknowledging (3); Working with the Child's Initiative and Agency (4); Using Language (7); and Using the Body (8).

\section{$\underline{\text { Insert box here }}$}

Danny had just settled at the play dough when his attention was drawn to another child, Jenny. Dawn watches with him (Strategy 1). He is then involved in balancing 
the rolling pin, which Dawn acknowledges (Strategy 3), 'Ooh look at that!', putting down the flattened dough which she had been holding for him.

Danny gives Dawn the rolled and folded dough. She acknowledges (Strategies 3 and 4), contextualises and extends his language (Strategy 7),

Danny: Roll it

Dawn: Roll it! Like a sausage roll.

She then offers him alternative directions that the imaginative play could take (Strategy 4),

Dawn: Thank you. Does it need cooking? Does it need to go in the oven? Or is it cooked already? [Pause]

Dawn: Shall I put it in the oven on a plate, or can I eat it now?

There is a pause in which Danny smiles and makes eye contact with Dawn. She interprets his use of his body verbally 'Can I eat it now?' and with the use of her own body (Strategies 3 and 8): sustaining eye contact; smiling; drawing in her shoulders in a complicit movement towards Danny; lifting and pretending to eat the roll. When she offers the roll back to Danny he meets it carefully with the rolling pin.

\section{The second part of the case study is Nina with Danny}

Nina interacted with her son, Danny (30 months), using the following Adult Pedagogic Strategies: Subtle Intervention (1); Acknowledging (3); Working with the Child's Initiative and Agency (4); Adult Learning (5); Using Language (7); and Using the Body (8).

\section{$\underline{\text { Insert box here }}$}

Nina is aware of how Danny is using his body pointing, curling and re-curling his fingers, to indicate his interests and explore objects, 'What are you showing me?' (Strategies 3 and 8). She responds to Danny's question about the treasure basket (Strategy 4). She acknowledges Danny's presence, emotions and capability by being physically close to him and letting him make contact with her hair with the brushes (Strategies 3 and 8). She also tunes into Danny's facial expressions and vocal intonation, including playfulness and teasing with the objects they find in the basket, such as the curtain rings and the metallic foil (Strategy 3). 
Danny: 'I like that one'

Nina: 'Yeah!'

Nina is working with Danny's initiative and agency (Strategy 4) checking his meaning and encouraging his curiosity and using language to support the child's actions, 'Noisy isn't it!' (Strategy 7).

Nina's timing is tuned to Danny's as they experiment looking through the rings (Strategies 1 and 5). Her movements are paced to match his as they explore using touch. Her close proximity and body position makes an intimate space for their interactions (Strategy 8).

Box ends here

\section{Discussion and involving fathers}

\section{The experience of the participants}

The project found many aspects of the new strategies resonated in our analysis of the episodes recorded with the male participants.

\section{The following example is of a parent, Darren and his reflection on 'Acknowledging' and 'Using the Body'}

Darren reflected on video episodes of his interactions with Oscar. He met with Penny, the researcher, and Sarah, Oscar's family worker. The video viewing discussion raised Darren's awareness. He recognised the use of touch in the family's own culture. He was prompted to record Oscar's older brother, Michael, caressing Oscar's head early in the morning.

Darren: I've seen it again with his hair and I put my finger out for his hand. Penny: Has anything surprised you?

Darren: I was surprised when we looked back at the video I noticed how many times I touched his hair

Penny: What do you think that's like for Oscar when you do that?

Darren: I think it's soothing for him to realise that I'm there and it gives him security that if he looks round I'm there.

Sarah: Like both touch and acknowledging together.

Penny: What about the value for you as the parent? 
Darren: I've noticed it around the house and at Dad's Group. I always seem to touch Oscar's hair all the time. Since we did the last video I've noticed it a lot more.

The use of video revealed surprising aspects of interactions for people who have worked in a setting or been a parent for years. A process of diffraction or transformation of perspective on what otherwise had been considered known occurred. Video case studies were, therefore, an appropriate tool for reviewing practice as Iedema (2014) found in his video reflections with medical practitioners and patients, "people often saw beyond what was displayed on the screen out across the organisation, back into the past, or forward into the future, linking what was shown to what was known' (2014,pg.198). The use of the body was identified through our raised awareness of multimodal interactions. Darren reconsidered what he knew of his own family culture in the light of the research discussion. However, Darren's finding does not correspond with Iedema's conclusion that,

while our multimodal analyses of interaction may identify bodily and behavioural peculiarities, these issues may not be easily communicated to those in the footage. Our findings may confront, precisely because they delve below the level of everyday consciousness, accessing the more embodied, and therefore normalised and naturalised aspects of existence (2014, pg.209).

Darren's realisation was his own, direct, readily explored and demonstrated in this research process. The participants' reflection on their practice showed that, while not all strategies are in use at any one time, the strategies were readily recognised by each of the participants as forming part of their own pedagogy. Similar processes of recognition were found through video reflection in Cremin, Burnard \& Craft's (2006) study of pedagogy in relation to fostering agency and possibility thinking.

The REPEY project noted some deficit in the responses of adults with regard to their own practice, 'The child-minders were not able to articulate explicitly their pedagogical understandings' (Siraj-Blatchford et.al., 2002, pg.113). By contrast the experiences of the participant parents in this research project indicated 
articulate and considered understanding of the findings and the implications for the community. Sue, Sally's mother, spoke of her experience. Regarding the themes that have emerged in the Pedagogic Strategies, I think the process is crucial to the development of the setting. I think we [parents] do all of this [strategies] all the time and through this [discussion] we learn the reasons'.

\section{The new strategies: acknowledging and using the body}

\section{Acknowledging}

Acknowledging relates to attunement, affirmation, accompanying, recognition, connection, and inter-subjectivity. Why should being noticed be an important part of these processes?

What is it about being noticed that is important? Is it simply that when we are noticed we notice that we have an impact on the world - that we are effective? Or is there something special about being noticed by other minds? (Reddy, 2008, pg.40).

Being noticed is fundamental for the child to interact and be involved and connect with others. Knowing that one has been noticed is the essence of inter-subjectivity, (Stern, 1985; Trevarthen, 1998). Inter-subjectivity in turn is the ground in which attachment grows (Stern, 1985). Acknowledging the child's presence is saying 'You're here'. There are arguments (Dalli et al., 2011a; Stern, 2004) to suggest that these moments of inter-subjectivity, of encounter between self and other, construct a sense of identity in the young child. We identified many instances of the adult noticing the child who approached him or her, and the child knowing that s/he had been noticed, that his/her presence was acknowledged.

Presence is both physical and emotional. Physically the adult could acknowledge presence in close proximity through touch or through creating an interactive-space (Payler, 2007) by the orientation of his/her body to the newly arrived child (this relates to the other new strategy - using the body).

The adult could also acknowledge over distance through speech, and through eye contact. It could happen without overtly intervening in the child's experience by 
active listening (Rinaldi, 2001), by using facial expressions and through alertness to the child's presence (Goodfellow, 2008). Acknowledging the physical presence does not happen only at the beginning of an interaction, but throughout an episode, the experience can be acknowledged from a child's perspective. For example, the family worker, Anna, asked one child who was watching the others on the slide, 'Can you see that, Henry?'

Beyond the physical presence and perspective of the child, the adult is also tuning into the emotional state and cognitive interest of the child responding to his/her expressions and actions. Acknowledgement is the foundation stone for attunement that is the key concept of quality identified in Dalli et al. (2011b), 'pedagogy with under-two- year-olds is realised in the establishment of attuned interaction between children and their caregivers who are present, supportive and responsive to the interactional cues of the infant and toddler' (2011b, pg.4). It is a strategy that does not require extensive resourcing or time. As Sam, another mother, reflected it was about, 'Presence, acknowledging presence as well as feelings. I didn't have to do a lot'.

As the fundamental basis for the learning relationship, and for the child's developing sense of identity, acknowledging as the initial part of inter-subjectivity could be the focus of significant further research, 'there are few investigations of the pedagogical role of the teacher in this area of under-two-year-olds' inter-subjective experience' (Dalli et al., 2011b, pg.76). Acknowledging could be identified in other cultures as connecting to the other person and as part of the inter-connectedness of learning, for example in the Maori context of 'whanaungatanga', where people are intimately connected to everyone else (Tamati, 2005).

Strategies are not completely separate. The categorisation only serves to clarify what is, in practice, interlinked. The acknowledging strategy underpins and complements the first 'Subtle Intervention' strategy. The English REPEY study also found that in the most effective pedagogy, shared purpose is founded on attunement to the perspective and knowledge of the child before intervening (Siraj-Blatchford et al., 2002, pg.725). We found the strategy of acknowledgement often co-occurred with the use of the body and touch, resonating with Johansson's description of intersubjectivity, 
A pedagogical encounter with the child's life world [...] involves approaching and trying to understand the child's whole being. Bodily experiences and expressions, as well as ways of relating to others constitute the components of a child's very existence in the world, and are as such significant for learning (Johansson, 2004, pg.11).

Acknowledging relates to the image we have of the child as a person and the stance we take to relating to that person, 'underpinning professional capabilities is a philosophy which describes an adult who seeks to connect with the essence of the child' (Atherton \& Nutbrown, 2013, pg.14). Acknowledging works within what Nutbrown calls 'a learner and person centred ethos' (2006, p. 125). Brennan (2005) calls this a 'culture of tenderness' when teachers help young children feel at home with love, patience, humour and personal attention. For Singer \& de Haan (2007) part of the teacher's role is creating a 'we- feeling' of togetherness. Greeting and giving attention to each child is a simple starting point for this relational pedagogy. In Elfer's $(1996,2007)$ research family interactions were described as 'intimate and spontaneous' and in setting interactions as 'more professional and planned' (2007, pg.169). Acknowledging and using the body raise questions about intimacy that perhaps need to be answered locally, at the setting level. Is there a place for more 'family-like' effective pedagogic strategies, intimacy and spontaneity in early years settings? When parents in this study were asked to rank the Pedagogic Strategies, they prioritised wanting their child to have acknowledging and touch and contact (using the body) when in the setting as that is what they would have had at home.

\section{Using the Body}

Young children learn using movement, physical actions, and tactile experiences. They have many modes of making meaning in addition to developing speech (Flewitt, 2006). In Multiple Intelligence Theory, Gardner (1983, 2006) sets out many forms of intelligence, whereas Malaguzzi (1998) thought intelligence is single but using many languages/modes of expression, both include using the body as part of an educational experience. An effective pedagogy will use and provide for the children using these many modes of participation (Bae, 2010). The project participants used touch at a finely controlled level, allowing their fingers to be guided by children to trace over the text and images on the page, or to explore the geometry of a watch-strap. They 
used their whole bodies making themselves into a base for the children, as a chair, a chaise lounge, a harbour, or platform.

\section{Insert photo here}

The adults also interacted using their whole bodies expressively as when Sarah skipped along a corridor with children. An embodied pedagogy, that integrates the body and mind, acknowledging the physical presence and experiences of the children and using the same modes of communication as they do, is called for internationally (Bresler, 2004; Chodarowski \& Egan, 2008; Hocking, Haskell \& Linds, 2001; O’Loughlin, 2006).

The pedagogic strategy using the body, like the Key Times training for 'the physical as professional' (Manning-Morton and Thorp, 2006) could ensure appropriate provision for the children rather than the alternative 'no touch' policies (Tobin, 2004) that neglect the needs of the children and, in our case, the preferences of parents. Developing good physical and mental health is supported through sensitive and responsive holding, and rocking, as well as embodied learning often in games (Tait 2007). Trevarthen $\underline{\&}$ Aitken (2001) suggest that physically rhythmic movements can be part of inter-subjective interactions encouraging emotional, cognitive and social growth if they are attuned. The way in which these physical interactions take place is significant for the child's positive sense of self (Gerhardt, 2004).

Safeguarding. One outcome of the project was to provide targeted safeguarding training for staff to support safe, healthy intimacy in interactions with children. Safeguarding can be seen in two different ways: keeping children safe; and safeguarding their well-being by ensuring that children have good experiences to flourish. Early childhood settings can combine these two important aspects, however, leadership needs to be equally confident and competent in both areas. Leaders must themselves be well trained and very clear about their robust policies, procedures and practices, and national statutory responsibilities (DfE, 2013).

\section{Implications, applications and limitations of the study}


The Pedagogic Strategies link to and complement other frameworks. In England they integrate with Positive Relationships used in guidance for the curriculum (Early Education 2012), and the professional standards for Early Years teachers (National College for Teaching and Leadership, 2013). Practitioners' workloads are subject to pressures in many early childhood contexts (DfE, 2014b, pg.6; Easthope \& Easthope, 2000). To introduce a framework for effective pedagogy in addition to the statutory requirements that practitioners fulfil would be an untenable proposition were the principles, practice and processes unrelated. Moreover, workload is not the only consideration of quality of experience for early years practitioners. Hargreaves and Hopper (2006) propose that the status of early years professionals would be increased by emphasising the pedagogical elements of their specialist expertise. Formosinho $\underline{\&}$ Figueiredo (2014) see the 'Pedagogy-in-Participation' approach as an asset rather than an imposition, and one that builds capacity for working with cultural diversity. The Pedagogic Strategies project has built staff capacity for working in the local community through appreciating cultural values and practices that have come from that locality.

The new revised framework of pedagogic strategies was agreed in whole staff meetings by the project setting staff. After this stage the pedagogic strategies framework was integrated into continuing professional development. It forms part of the peer-peer observation review process for all practitioners working with under and over threes. The Early Years Inspection Handbook (Ofsted, 2015) has a strong emphasis on observation of young children and practitioners. During the inspection process the inspector will focus on how well adults care for children and consider the impact of what the adults do on children's learning and development. Inspectors are required to make one or more joint observations to gain an insight into the effectiveness of the provision's professional development programme for practitioners. The Pedagogic Strategies approach will support practitioners in responding to Ofsted inspector's questions and lines of enquiry about how they are ensuring and developing quality early years education in their setting. See chapter three for more information about peer-peer reviews.

\section{What did we learn?}


There have been extensive reviews of what constitutes quality in early years pedagogy. What is distinctive about this project is the parent and practitioner developmental partnership co-constructing strategies that are local, relevant and owned by the participants. The findings show a high degree of consistency with the findings of the major studies. This project recognised the extensive pedagogy that parents have with their children and identified two strategies that are particularly important for children under three: acknowledging and using the body. Although there is a need for further research into the pedagogical role of the educator in intersubjectivity, this project demonstrates how acknowledging interweaves with the use of touch in safe, healthy intimacy with children, and more broadly shows the value of an embodied pedagogy. The project team would recommend that all early years staff teams have opportunities to enquire into their own pedagogy, the values and priorities in their settings, and that they should do this with the families in their community.

\section{$\underline{\text { Word count } 6083}$}

